\title{
Capacity Modelling for Virtual Enterprises
}

João A. Bastos (1), Jorge P. Sousa (1,2)

(1)INESC - Rua José Falcão, 110 - 4300 Porto, Portugal

(2)FEUP - University of Porto

email: \{jbastos, jsousa\}@inescn.pt

\begin{abstract}
The purpose of this paper is to present a new view on the problem of capacity planning in a distributed network of enterprises, i.e. in a Virtual Enterprise (VE). This view is based on a distributed planning architecture, that takes into account the requirements and characteristics of local capacity models.

In order to cope with the new emerging problems that the VE are encountering, we have been designing and investigating two generic architectures of Decision Support Systems (DSS) for VE planning with capacity checking, applicable to different scenarios of distributed networks of enterprises. The first architecture is hierarchical and is based on the existence of a global decision-maker. The second one is fully distributed and is based in a multi-agent co-operative environment. This work is part of a broader project with a particular focus on the semiconductor industry.
\end{abstract}

\section{Keywords}

Virtual Enterprise, Capacity Modelling, Decision Support Systems, MultiAgents 


\section{INTRODUCTION}

The purpose of this paper is to present a new view on the problem of capacity planning in a distributed network of enterprises, i.e. in a Virtual Enterprise (VE). This view is based on a distributed planning architecture, that takes into account the requirements and characteristics of local capacity models. The paper presents part of the authors' work and results on the ESPRIT project X-CITTIC - A Planning and Control System for Semiconductor Virtual Enterprises (E.20544).

Semiconductor manufacturing and other high technology industries are facing competitive pressures from the market, the customers and the way enterprise operations are carried out. The whole business process effectiveness demands the set-up of networks of enterprises, with efficient planning and control of the order flow (Richards et al.,1997).

In general terms, we might say that the main goal is to produce a "feasible" production plan for the whole VE, satisfying due dates, with the minimal overall cost. In order to achieve this goal, on each node of the network, a hopefully accurate, updated and reliable local capacity model must be available.

The current trend for grouping together heterogeneous and complementary enterprises with common interests, poses several questions that require solutions compatible to the several interests in presence. These political and economic relations between units belonging to the same $\mathrm{VE}$ are currently ranging from a very centralised authority (based on the power of a core company) to more democratic and decentralised organisations (based on units of the same economical importance to the VE).

When we face these scenarios, the problem domain becomes quite complex and broad. Nevertheless the approaches presented in this paper try to encompass both situations, tackling three of the main concerns involved in capacity modelling of a Virtual Enterprise: the distribution of the information through several enterprise units, the huge volumes of the information involved and the resolution of conflicts.

\section{THE VIRTUAL ENTERPRISE ENVIRONMENT}

Within the Virtual Enterprise, manufacturing products may involve many different and geographically distributed sites, with several suppliers and external subcontracted companies, that are connected to each other by sharing information via e.g. an electronic network, namely the Internet. In order to be sufficiently agile and achieve the desirable performance, the production chain has to be well coordinated by efficient and effective planning at the VE level (Ross, 1997).

In this environment, it is crucial to provide fast and on-time responses when dealing with customer inquiries and the respective order commitments. Today's business practices impose fast and accurate responses to customer requests, namely concerning customer delivery dates, as the seller's reputation on "deliveries on time» is an important issue in the supplier/customer relationship.

In particular, the semiconductor manufacturing industry is a good example of the major trends on the distributed enterprises network domain. Moreover, as this 
industry is a capital-intensive business under strong competition, companies constantly face a need to have accurate delivery dates.

To cope with the new emerging problems that the VE (in general) are encountering, we have been designing and investigating two generic architectures of Decision Support Systems (DSS) for VE planning with capacity checking, applicable to different scenarios of distributed networks of enterprises.

We first consider a two layer hierarchical architecture (see figure 1). On the top level, we have a global planning component, dealing with very aggregate information. This component processes the inquiries from the customers, and after a consultation to the local capacity models (bottom layer) assigns work orders along time to local production sites, according to the production objectives and constraints, and trying to minimise costs. Within this high level planning activity, we have the logistics issues. On the bottom level, for each site we have local components modelling each of the local units of the VE. These local capacity models are based on local information and can be designed with various levels of detail. Their main purpose is to respond to the global planner requests by providing feasible dates and the associated costs. With this architecture, the issue of information distribution and the volume problem are resolved in a hierarchical manner. This is accomplished with a central global component that deals only with globally aggregated information and with several local capacity models spatially distributed that achieve their own purposes by using more detailed local data.

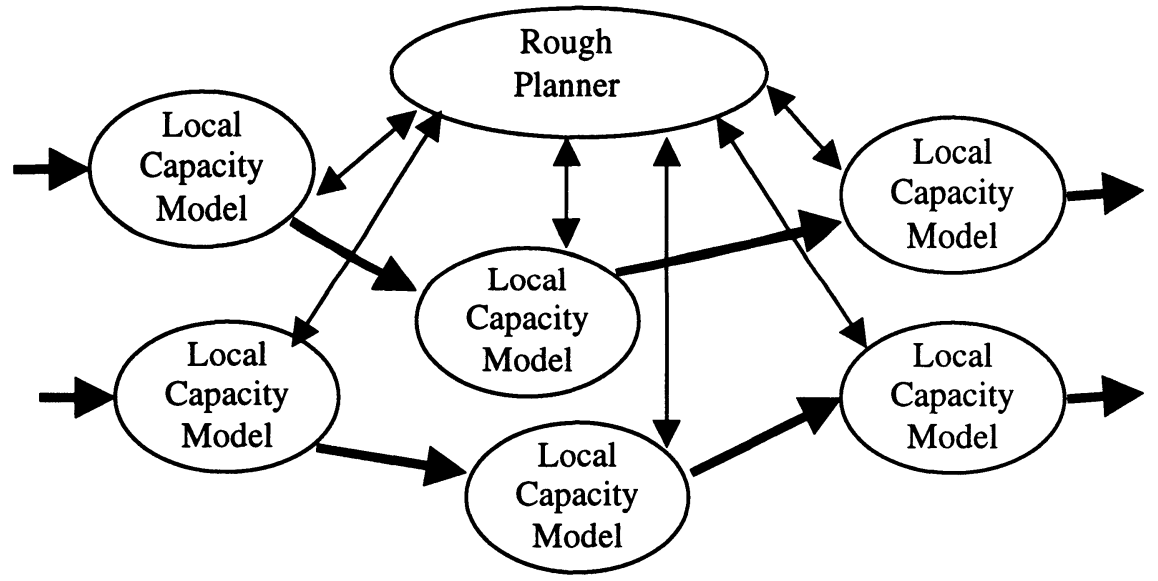

Figure 1- Hierarchical Architecture

The second architecture is fully distributed, and is based on a multi-agent cooperative environment (see figure 2). On each site, we still have a local capacity model with its own specific implementation, but on top of it, there is now a new functionality obtained through a new layer, providing this component with the capability to negotiate with other local components. This agent view offers a level of abstraction in which the algorithmic and computational systems interoperate 
globally across the network, linking the several nodes of the VE. With these autonomous, goal-oriented local agents, it is possible to perform complex negotiations and try to achieve co-ordinated solutions, hopefully close to what might be called the global optimal solution. With this architecture, mediation and negotiation between all the agents tackle the issue of the information distribution. On the other hand the volume of information involved in the agents reasoning and constraint propagation is reasonably small, since each agent, even if it is using very detailed information, communicates with each other through a high level language, leading to a compact level of data exchange.

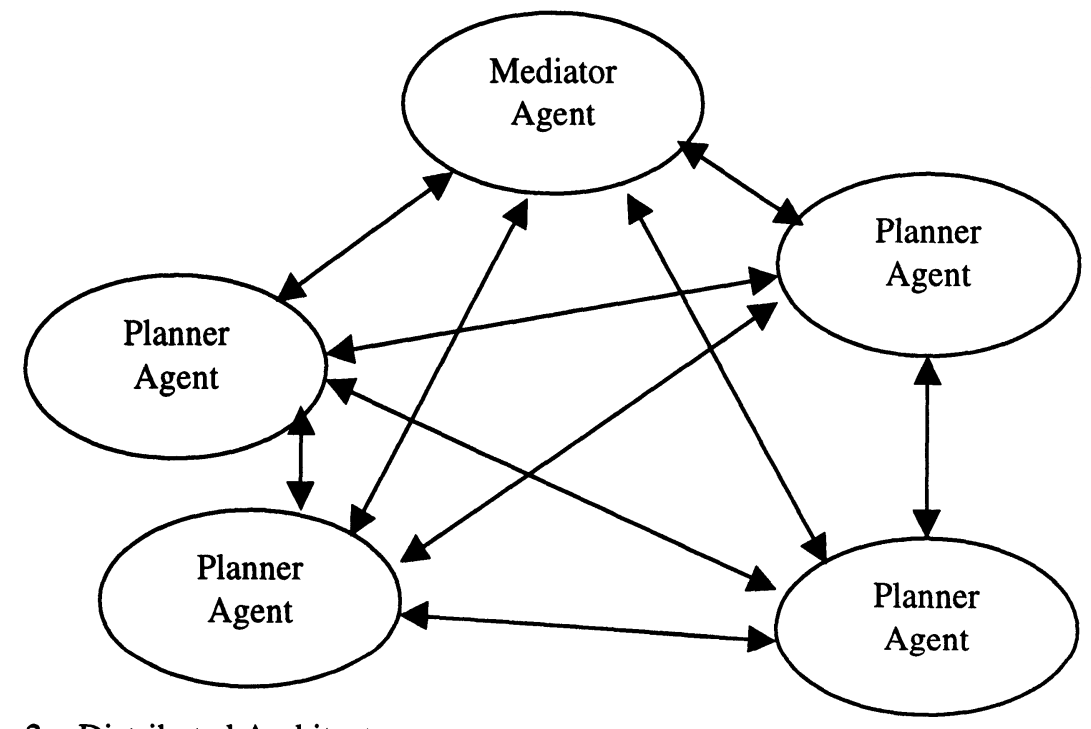

Figure 2 - Distributed Architecture

\section{THE HIERARCHICAL ARCHITECTURE}

The hierarchical architecture is based on the existence of a global decisionmaker. This component deals only with very aggregate information and its main function is to efficiently co-ordinate production, keeping the most intensive information processing and calculations on the local systems, and enforcing that these operations are performed in parallel, speeding up the search for solutions.

The global planning component, upon receiving a customer inquiry, starts by determining which VE units are candidates for production. Then, several consulting requests are sent to those candidate units, with proposals stating tentative dates for production. The local units will respond, by saying whether those proposals are feasible or unfeasible, and eventually by producing counteroffers. The next task of the global component is to test the global feasibility of the solutions sent back by the local components, evaluate their overall costs and if a "good solution" is not achieved, initiate another consulting iteration with new trial dates. This iteration process continues until a "good solution" is achieved and a proposal can be presented to the customer. The main objective of the global 
planning algorithm is to obtain this "good solution", minimising the overall costs in a small number of iterations. These solutions can be described as stage routes with start and finish dates, and quantities for each (see figure 3 ).

Currently, we are designing and evaluating several algorithmic approaches based on randomised local search procedures, namely genetic algorithms, simulated annealing and taboo search.

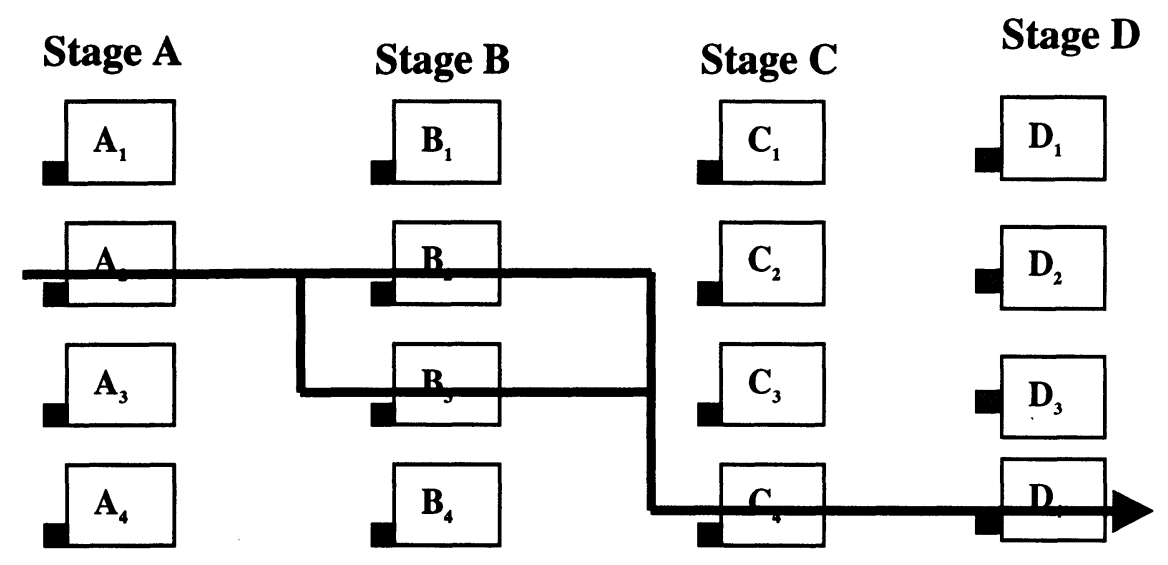

Figure 3 - Partial representation of a solution

The local planning systems deal with capacity resources, order loads and time periods, and have a generic interface between the data structures of the capacity model and the "planning engine" (the algorithm). This structure allows that different planning engine algorithms can be instantiated and easily connected to the other elements of the planning component. Several algorithms are currently under test and evaluation, and they stand from simple allocation algorithms (such as forward or backward scheduling) to more elaborated and complex methods such as dynamic bottleneck search.

Obviously each site that belongs to the VE is singular. The role of each capacity model is exactly to capture these specific features, its main functionality being to:

- Represent each site capacities in an easy to integrate software model;

- Evaluate customer inquiries through a centralised Rough Planner;

- Insert any new local order in the capacity model and pass it to the local scheduler.

\section{Structure of the Capacity Model Component}

The Capacity Model (see figure 4) is composed by several blocks with the following functionality:

- Capacity Model main block - interconnects with the other blocks and is the core of the component as it uses the static and dynamic data to build a capacity data model; 
- IDL interfaces with IM or RP - interchange data with other components and send and receive events and signals;

- Graphical User Interface - used to configure, tune and monitor the behaviour of the capacity model;

- Algorithm - this is a "plug and play» block that is inserted in the capacity model according to the reality we want to model.

\begin{tabular}{|c|c|c|}
\hline $\begin{array}{c}\text { IDL with } \\
\text { Information } \\
\text { Manager }\end{array}$ & \multirow{2}{*}{$\begin{array}{c}\text { Capacity } \\
\text { Model }\end{array}$} & GUI \\
\cline { 1 - 1 } $\begin{array}{c}\text { IDL with } \\
\text { Rough Planner }\end{array}$ & & Algorithm \\
\hline
\end{tabular}

Figure 4 - Structure of the capacity model component

\section{Algorithms}

The system architecture was designed in order to accommodate different algorithm implementations and instances. These instances can range from simple forward and backward strategies to queue theory based procedures or local search heuristics. Currently, we are successfully testing an approach based on bottlenecks as capacity constraints.

The bottlenecks choke the flow of materials in the factory and dictate the final throughput. Therefore they need to be fully utilised in order to maximise the resource utilisation.

In a first stage, bottleneck optimisation focus on the bottlenecks and try to optimise their operation. Then, for the operations that are before the bottleneck, it performs a backward schedule allocation, and for those after the bottleneck, a forward schedule is used.

The main difficulty of this approach is how to know what are the bottleneck resources prior to the full load of the model. This is due to the fact that the capacity model does not know the full load of orders that are going to be inserted in the future (since they are inserted sequentially as they arrive online).

The solution we have implemented is based on a simple concept, the dynamic bottleneck detection. This mechanism works as follows: at the beginning, an expert planner is asked to define a default bottleneck and also bottleneck candidates. After this configuration is completed and the RCM component is initialised, the capacity model is ready to respond to inquiries and to insert new orders. To perform these tasks, the model uses the default bottleneck resources. Therefore, when the component receives a new order to insert, it loads the default bottleneck and uses its capacities to make any offer for inquiries. In the meantime, the algorithm loads each of the bottleneck candidates in a faster and more aggregate way.

When the model reaches a near complete load state, the dynamic bottleneck detection mechanism cheeks if any of the bottleneck candidates has reached the condition of actual bottleneck of the system, for any of the specific periods of time, 
and if its load is larger than the current default bottleneck. If this condition is met, the current default bottleneck is replaced. This action means that for the next inquiry or order insertion the resource capacity used will be computed based on this new default bottleneck. Therefore, this procedure performs a continuous online seek of the bottlenecks for all the periods of the planning horizon.

Finally, it should be noted that the user plays a major role in this process, by interacting with the models through graphical interfaces (GUI). A first GUI is concerned with the static configuration of the model, namely with the definition of the default and bottleneck candidates and with the definition of the level of data aggregation used in the construction of the model. A second GUI is used dynamically during run-time as a monitoring tool of the behaviour of the capacity model and as a fine tuning mechanism of that model.

Several capacity models have been developed and implemented and are under evaluation by the industrial partners of the X-CITTIC project. The approach is very promising, and with the user's suggestions, new interesting research ideas are under study to improve these capacity models.

\section{THE DISTRIBUTED ARCHITECTURE}

The current business trends are forcing industrial enterprises to form temporary associations or networks leading to the so-called Virtual Enterprises. These VE are becoming more and more volatile and dynamic due to the market pressures. As a consequence, the aggregation element of this enterprises is now less related with a Core Enterprise that aggregates several companies in a VE, but more with a business concept based on common interests. This environment creates a framework for fully distributed VE, where each unit is more aware of its rights and obligations and no longer accepts certain forms of centralised business domination.

Therefore for a fully distributed VE, the centralised architecture is no longer feasible. Consequently, new approaches are required. Among theses approaches is the multi-agent co-operative and distributed architecture.

With this multi-agent architecture, based on co-operating intelligent entities that make decisions through negotiation, it is possible to perform the same tasks of the centralised co-ordination approach, in a distributed manner. Essentially the core of the local planning components is the same as in the hierarchical architecture. But on top of these computational components, a new layer is added. This layer offers an agent view to the external environment of the component and provides a level of abstraction in which the co-ordinating behaviour is available.

In this architecture, an agent is a software component that can perceive its environment through sensors and act upon that environment through effectors (Russel and Norvig, 1995). In our case study, these agents are software components that try to generate a «plan» for the entire VE through negotiation. The way these agents «perceive» their environment is by message exchange between them. The interaction between the agents is represented in figure 5 . 


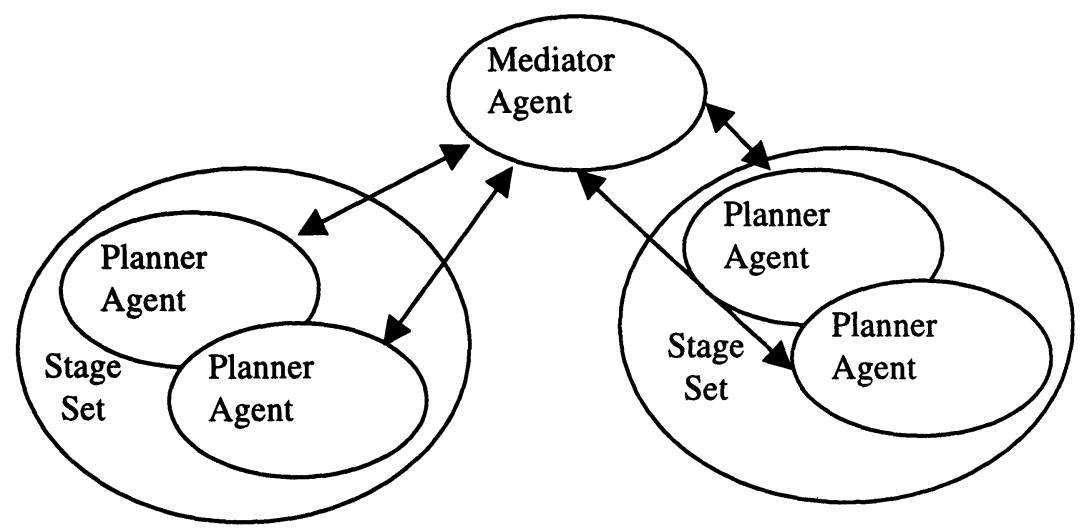

Figure 5 - Multi-agent distributed architecture

The mediator agent has the role of interacting with the customers and of sending order inquiries and order confirmations to the network of planning agents. The planning agents are themselves organised in sets of production stages. This positioning means that each planning agent belonging to a stage set is competing with the other agents of the same set for the same jobs. Although each planning agent represents a unit on the VE, if a specific enterprise or site can perform more than one production stage, this unit will have a number of planning agents equal to the number of production stages.

\section{Components of the fully Distributed Planning System}

All agents communicate by using a high level language and sharing the same internal representation.

- Mediator Agent - This agent has the structure represented in figure 6. The GUI supports the interaction with the user. The communication interface allows the communication with the planning agents of the VE. The mediator system is the core of the planning mediator agent and is responsible for receiving and processing any customer inquiry, receiving the results of the negotiation, compiling them to send them to the customer, and mediating conflicts when they arise between the planning agents.

\begin{tabular}{|c|c|c|}
\hline GUI & $\begin{array}{c}\text { Mediator } \\
\text { System }\end{array}$ & $\begin{array}{c}\text { Communication } \\
\text { Interface }\end{array}$ \\
\hline
\end{tabular}

Figure 6-Mediator agent 
- Planning Agent - This is a local agent with a capacity model and a reasoning mechanism (see figure 7). The planning agent is essentially a capacity model with a planning algorithm, and an intelligent layer on top of it with capacities to negotiate and collaborate, to attain good global solutions for the global planning problem.

\begin{tabular}{|c|c|c|c|}
\hline $\begin{array}{c}\text { Communication } \\
\text { Interface }\end{array}$ & $\begin{array}{c}\text { Planner Agent } \\
\text { System }\end{array}$ & $\begin{array}{c}\text { Capacity } \\
\text { Model }\end{array}$ & GUI \\
\cline { 4 - 4 } & & $\begin{array}{c}\text { Planner } \\
\text { Algorithm }\end{array}$ \\
\hline
\end{tabular}

Figure 7 - Planning agent

\section{Distributed Algorithm}

The main objective of this distributed architecture is to create a domain in which autonomous agents work for individual and common goals, in a shared environment. Co-ordinated goal seeking is performed by using a constraint based problem-solving model. As each individual agent is a constraint based problem solver, the agent interactions are therefore composed by the creation, the deletion and the exchange of constraints (Beck, 1994). Agents constrain each other through explicit time and product quantity constraints that are passed and evaluated up and down stream.

The generation of an offer to a customer inquiry naturally starts when a customer sends a request to the mediator agent. This agent then sends an inquiry request to all of the final production stage planning agents registered to him.

From this point on, a double step procedure is initiated. First, a generation procedure is performed to get a first solution; and afterwards, a negotiation and cooperation procedure is performed in order to improve the initial solutions and try to optimise the offer to be sent to the customer.

\section{Generation Phase}

The generation phase produces initial solutions that can be improved in further steps. These initial solutions are obtained through a kind of backward scheduling procedure as follows (see figure 8):

- After the last stage planning agents receive the customer inquiry request, they generate quantities and finish dates for the previous stage, that are passed to all the planning agents of the previous production stage.

- The previous or upstream production stage planning agents receive these constraints (finish dates, quantities and last stage site costs) and evaluate them, generating also constraints (finish dates, quantities and costs) for the previous or upstream production.

- The procedure continues until the first production stage is reached and each planning agent of the first stage generates an offer (with a stage production route) based on the previous downstream stages.

These offers are still not optimised and can be an infeasible solution (for instance 
the start date can fall before the present day).

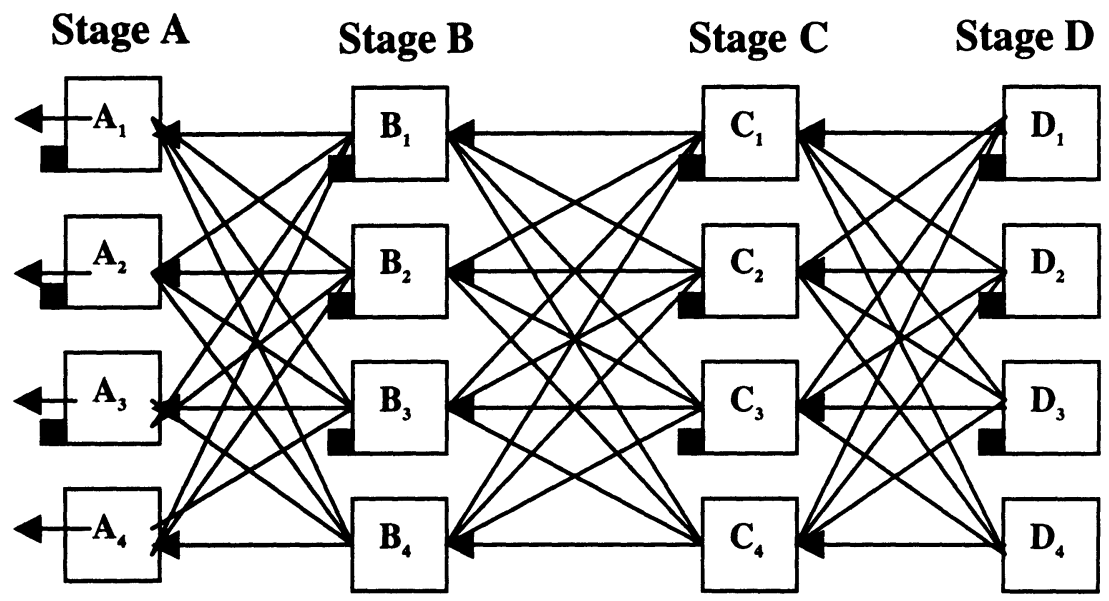

Figure 8 - Generation phase: creation of initial solutions

\section{Negotiation and Co-operation Phase}

After the initial generation phase, a more elaborated procedure starts in order to improve the initial solutions. These initial solutions have been produced through a greedy approach from each of the planning agents involved, interested in searching for its own local optimum. To find better solutions, closer to the global optimum, a phase of negotiation and co-operation is started:

- Negotiation - The first stage planning agents, based on the initial solutions, produce and send to all the second stage planning agents, constraints which are then evaluated and generate other constraints to the next stage, and so on. This constraint wave reaches the final stage and inverts its direction, moving towards the first stage. This movement goes on until we reach a satisfactory solution or a point where no improvement is obtained (see figure 9). 


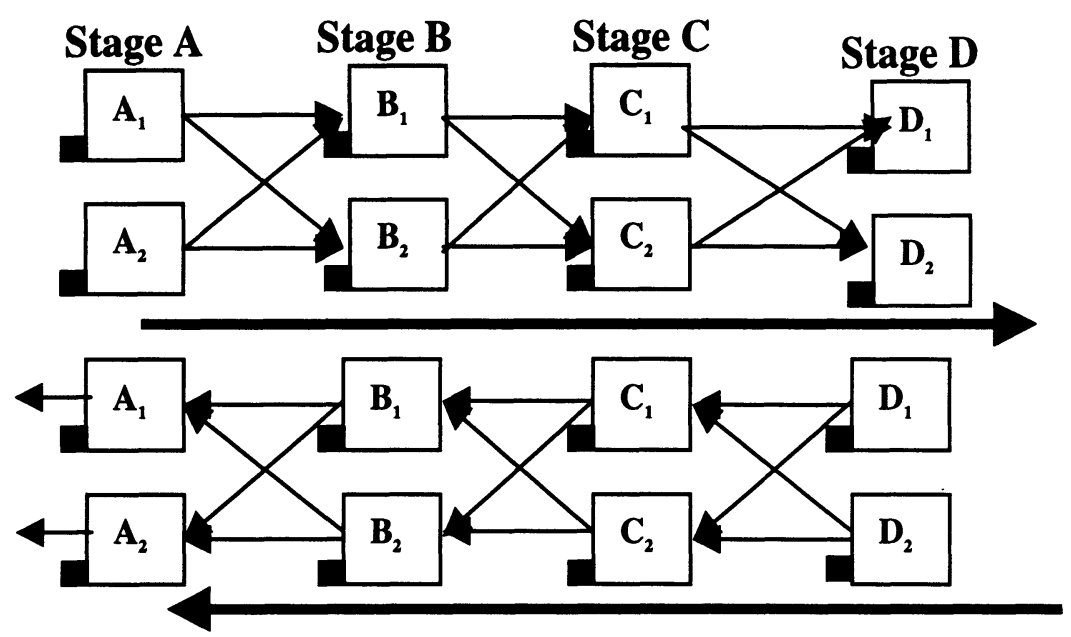

Figure 9-Negotiation: improvement of initial solutions

- Co-operation - At each stage, in parallel with the negotiation process, an interaction with the other planning agents of the same stage takes place, eventually leading to order splitting (division of the quantities to produce, among different sites in the same stage). This may in certain cases, be an interesting solution (see figure 10).

As a result of this negotiation and co-operation wave, hopefully better global solutions are produced, and sent to the mediator agent. The mediator agent presents these solutions to the customer as alternatives or chooses the «best» one (based on cost and start and finish dates satisfaction).

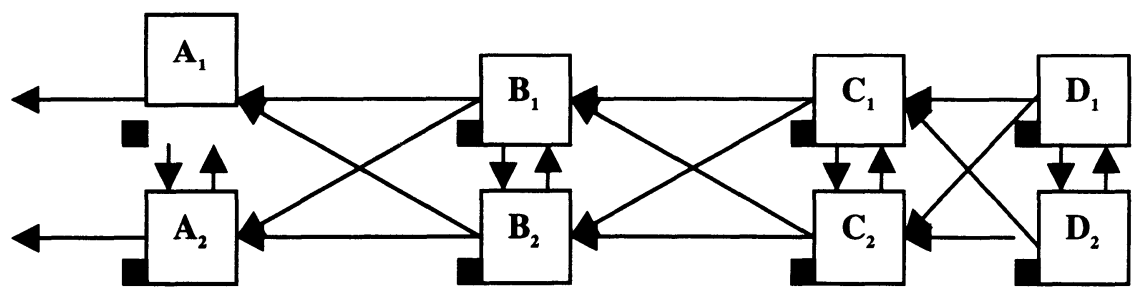

Figure 10 - Co-operation: order splitting within a stage

\section{Final Considerations}

The capacity models in this distributed architecture are the same as in the hierarchical architecture. The main difference of this approach is on the intelligent layer on top of the capacity models, that integrates these new agents in a cooperative environment.

In the process of co-ordination, conversations between dependent agents are 
performed and through constraint relaxation, a "global good solution" is searched. These conversations are carried out according to a set of previously agreed rules, and the way conflicts are resolved is based on constraint relaxation logic (with the most weighting factors being costs and due dates).

This distributed architecture is still under evaluation and the constraint-based problem solving methodology is currently a subject of research and experimentation. We are also working on improving the communication between agents, to get more efficiency in the search for solutions of the planning problems. This effort will also provide each agent with enhanced capabilities to support agent assumptions about the other agent's internal states.

\section{CONCLUSIONS}

In this paper we have presented a new view on the problem of capacity planning in a Virtual Enterprise (VE). This view was based on two generic architectures of Decision Support Systems (DSS) for VE planning with capacity checking, applicable to different scenarios of distributed networks of enterprises. The first architecture is hierarchical and is based on the existence of a global decisionmaker. The second one is fully distributed and is based in a multi-agent cooperative environment.

When comparing the two architectures, it seems clear that the hierarchical approach is simpler and more efficient in fast achieving feasible and satisfactory solutions. However these solutions do not fully take into account many concerns and objectives of the local units. Its main drawback is due to the fact that the global decision centre is located on a specific site, and on a loosely connected VE this could be a political problem. In such cases, the distributed architecture is more suitable, since there is no centralised decision centre, and each site has the same opportunities on the "planning negotiation". Moreover, on each multi-agent scenario, it is possible to "optimise" the negotiation process, by implementing practical rules, already successfully used in the VE, or eventually by experimenting new rules.

In summary, we might say that both architectures have a considerable potential, and may become important tools in supporting the planning processes of distributed networks of industrial enterprises. The precise scope of these approaches is a key research topic we are currently addressing.

\section{REFERENCES}

Beck, J. (1994) A Schema for Constraint Relaxation with Instantiation for Partial Constraint Satisfaction and Schedule Optimisation (M.Sc thesis, University of Toronto).

Russel, S and Norvig P. (1995) Artificial Intelligence - A modern Approach. Prentice-Hall.

Richards, H. D., Dundenhausen, H. M., Makatsoris, C. and de Ridder, L. (1997) Flow of orders through a virtual enterprise - their proactive planning and 
scheduling, and reactive control. Computing \& Control Engineering Journal, August 1997, 173-9.

Ross, D. F. (1997) Competing Through Supply Chain Management. Chapman \& Hall.

\section{BIOGRAPHY}

João Bastos obtained the degree of Mechanical Engineer at the Faculty of Engineering of the University of Porto and is doing research work at INESCUESP. He holds a MSc. in Industrial Informatics and is currently undertaking his $\mathrm{PhD}$ work in the area of intelligent manufacturing systems.

Jorge Pinho de Sousa is an Assistant Professor at the Faculty of Engineering of the University of Porto and researcher at INESC-UESP. He holds a Ph.D. in Operations Research (Université Catholique de Louvain, Belgium, 1989). His main interests are on Operations Research, Combinatorial Optimisation, Project Management, Industrial Organisation, and in Production and Operations Management. 